
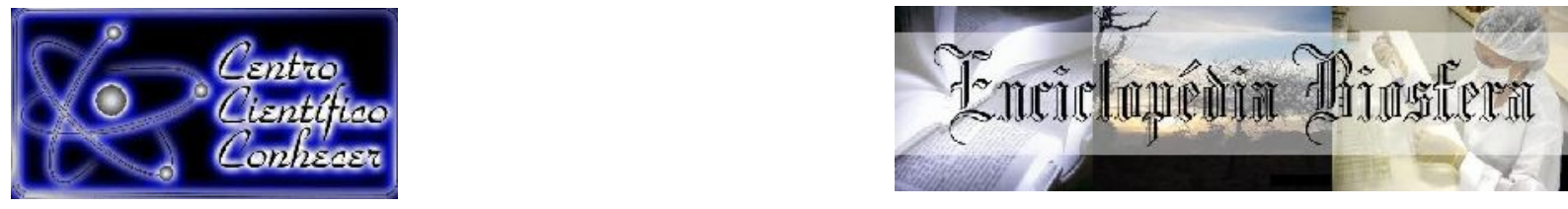

\title{
METABÓLITOS SECUNDÁRIOS DE PLANTAS MEDICINAIS USADAS EM GARRAFADAS POPULARES COMERCIALIZADAS EM FEIRAS LIVRES DE BELÉM, PARÁ, BRASIL
}

\author{
Ana Flávia Santos de Brito ${ }^{1 *}$, Altem Nascimento Pontes ${ }^{2}$ \\ ${ }^{1}$ Graduação em Ciências Naturais - Biologia, pela Universidade do Estado do Pará \\ (UEPA) \\ ${ }^{2}$ Docente do Programa de Mestrado em Ciências Ambientais da Universidade do \\ Estado do Pará (UEPA) \\ *Autor para correspondência: anabritosalvatore@gmail.com
}

Recebido em: 15/05/2021 - Aprovado em: 15/06/2021 - Publicado em: 30/06/2021

DOI: 10.18677/EnciBio_2021B15

\begin{abstract}
RESUMO
Utilizadas para várias finalidades na medicina popular brasileira, as garrafadas, de modo geral, são feitas por soluções constituídas principalmente por dois ingredientes, a bebida alcoólica ou não alcoólica e a combinação de plantas medicinais. Muitas vezes, as garrafadas geram desconfiança para algumas pessoas que não têm o hábito de consumir o produto, mas têm curiosidade de experimentar, devido ao fato de não serem explicadas suas composições medicinais claramente nos rótulos. O presente artigo buscou conhecer as variedades de garrafadas medicinais mais populares comercializadas nas feiras livres de Belém, bem como comparar o valor científico da composição das plantas comumente usadas nas garrafadas com a utilidade destinada a elas por meio de literaturas científicas. Foram analisados os rótulos de 23 garrafadas das principais feiras livres de Belém. A partir desses rótulos, foram listadas e tabuladas as indicações para uso e os dados da composição de plantas medicinais das garrafas, através dos sites Flora do Brasil e Trópicos. Posteriormente, comparadas com a literatura etnobotânica sobre a composição dos metabólitos com atividades biológicas de certas plantas. Os resultados obtidos revelaram uma grande diversidade de espécies entre as garrafadas, além do compartilhamento de garrafadas com mesmas espécies. Foi possível constatar a quantidade de plantas que compõem as garrafadas, e plantas com alta quantidade de metabólitos com atividade biológica. Dessa maneira, evidenciando-se o valor científico das garrafadas através do potencial de plantas com propriedades medicinais.
\end{abstract}

PALAVRAS-CHAVE: Garrafadas Medicinais. Saber científico. Saber popular. 


\title{
SECONDARY METABOLITES OF MEDICINAL PLANTS USED IN POPULAR BOTTLES COMMERCIALIZED IN FREE FAIRS IN BELÉM, PARÁ, BRAZIL
}

\begin{abstract}
Used for various purposes in Brazilian popular medicine, bottles are generally made up of solutions consisting mainly of two ingredients, alcoholic or non-alcoholic beverage and the combination of medicinal plants. Often bottles create suspicion for some people who are not in the habit of consuming the product, but are curious to experiment, due to the fact that their medicinal compositions are not clearly explained on the labels. The present article sought to know the most popular medicinal bottlenose varieties marketed in the fairgrounds of Belém, as well as to compare the scientific value of the composition of the plants commonly used in the bottles with the utility destined to them by means of scientific literatures. The labels of 23 bottles of the main Belém free trade shows were analyzed. From these labels, the indications for use and the data of the composition of medicinal plants of the bottles were listed, through the sites Flora do Brasil and Tropics. Later, compared with the ethnobotanical literature on the composition of the metabolic with biological activities of certain plants. The results obtained revealed a great diversity of species among the bottles, besides the sharing of bottles with the same species. It was possible to verify the number of plants that compose the bottles, and plants with high amounts of metabolic activity with biological activity. This way, evidencing the scientific value of the bottles through the potential of plants with medicinal properties.
\end{abstract}

KEYWORDS: Medical Bottles. Popular knowledge. Scientific knowledge.

\section{INTRODUÇÃO}

Segundo a definição do Anteprojeto de Lei sobre o acesso a Recursos Genéticos, Conhecimentos Tradicionais e Repartição de Benefícios (BRASIL, 2015), o conhecimento tradicional é todo conhecimento, inovação ou prática de comunidade tradicional relacionado aos componentes da diversidade biológica. Um exemplo desse conhecimento tradicional empregado ao uso das plantas medicinais são as garrafadas comercializadas em feiras livres e mercados populares (CAMARGO, 2011). Utilizadas para várias finalidades na medicina popular brasileira, as garrafadas, de modo geral, são feitas por soluções constituídas principalmente por dois ingredientes: a bebida alcoólica ou não alcoólica e a combinação de plantas medicinais (PASSOS et al., 2018).

As garrafadas geram desconfiança para algumas pessoas que não têm o hábito de consumir o produto, mas têm curiosidade de experimentar, devido ao fato de não serem explicadas suas composições medicinais nos rótulos das garrafas em que são vendidas (PREFEITURA DE BELÉM, 2017). Ainda, algumas apresentam em seus rótulos apenas certos nomes vulgares, sendo a procedência das plantas desconhecidas. Por se tratarem de uso tradicional e cultural não é levada em consideração a necessidade de especificação da composição do produto (PASSOS et al., 2018).

As garrafadas não podem ser confundidas com fitoterápicos, pois estes são medicamentos obtidos empregando-se exclusivamente matérias-primas ativas vegetais, além disso, um fitoterápico é caracterizado pelo conhecimento da eficácia e dos riscos de seu uso, assim como pela reprodutibilidade e constância da qualidade (BRASIL, 
2006). A eficácia e segurança, segundo a Agência Nacional de Vigilância Sanitária (ANVISA, 2004), são "validadas através de levantamentos etnofarmacológicos de utilização, documentações tecnocientíficas em publicações ou ensaios clínicos".

As garrafadas também não podem ser chamadas de medicamentos, pois medicamentos são "produtos farmacêuticos, tecnicamente obtidos ou elaborados, com finalidade profilática, curativa, paliativa ou para fins de diagnósticos" (BRASIL, 1973). Desse modo, é "uma forma farmacêutica terminada que contém o fármaco, geralmente, em associação com adjuvantes farmacotécnicos" (ANVISA, 2004). As garrafadas então possuem como principal embasamento teórico, o próprio conhecimento popular ou local (SOUZA, 2011).

No mercado do Ver-o-Peso, em Belém, capital do estado do Pará, para preservar o segredo da fórmula, algumas erveiras admitem não colocar no rótulo da garrafa todos os ingredientes contidos no recipiente (PREFEITURA DE BELÉM, 2017). E dizem ainda, "Em alguns casos podem ser até 20 folhas de plantas medicinais" ou "raízes diferentes". Segundo Passos et al. (2018), não existe uma regulamentação da ANVISA para esse tipo de produto, logo não são submetidas a nenhum teste de segurança, eficácia e qualidade.

Embora a medicina moderna esteja bem desenvolvida na maior parte do mundo, a Organização Mundial da Saúde (OMS) reconhece que grande parte da população dos países em desenvolvimento depende da medicina tradicional para sua atenção primária, tendo em vista que $80 \%$ desta população utilizam práticas tradicionais nos seus cuidados básicos de saúde e $85 \%$ destes utilizam plantas medicinais ou preparações destas (BRASIL, 2006).

Algumas dúvidas e curiosidades ficam explícitas quando se fala em garrafada (PASSOS et al., 2018). Pensando nisso, conhecer a procedência das plantas medicinais usadas na medicina popular, caseira e cultural, como, por exemplo, em garrafadas, diminui a desconfiança daqueles que têm curiosidade em experimentar o produto e para isso é importante tomar conhecimento sobre as plantas com atividades biológicas é importante (FRANÇA et al., 2007).

Desse modo, os objetivos desse artigo foram conhecer as variedades de espécies de plantas medicinais das garrafadas mais populares comercializadas nas feiras livres de Belém, além de comparar as propriedades científicas das plantas mais representativas usadas nas garrafadas com a utilidade destinada a elas por meio de literaturas científicas. As questões norteadoras deste projeto foram: Quais espécies de plantas medicinais são comumente usadas nas garrafadas e qual sua identificação científica/taxonômica? Há quais famílias botânicas pertencem essas espécies? O uso indicativo nos rótulos das garrafadas está de acordo com o potencial da composição científica daquelas plantas medicinais já descritas na literatura? Quais são os metabólitos com atividades biológicas das plantas medicinais mais usados nas garrafadas?

\section{MATERIAL E MÉTODOS}

O estudo foi realizado na cidade de Belém, capital do estado do Pará, localizada no nordeste do Estado, a $120 \mathrm{~km}$ do mar e $160 \mathrm{~km}$ da linha do equador, conforme Figura 1. Possui aproximadamente 1.059,458 $\mathrm{km}^{2}$ (IBGE, 2010) de área territorial, sendo capital do Pará. Fundada no dia 12 de janeiro de 1616, a cidade foi a primeira 
capital da região Norte do Brasil. Banhada pelo rio Guamá e pela Baia de Guajará, é quase uma península com apenas uma via de acesso de entrada e saída que é a BR316.

Belém tem aproximadamente 1.393.399 habitantes (IBGE, 2010), a economia baseia-se primordialmente nas atividades de comércio e serviços, embora seja também desenvolvida a atividade industrial com grande número de indústrias alimentícias, navais, metalúrgicas, pesqueiras, químicas e madeireiras (PREFEITURA DE BELÉM, 2017).

FIGURA 1. Mapa de localização das feiras visitadas no município de Belém. 1- Feira da Bandeira Branca; 2- Feira do Guamá. 3- Feira do Jurunas; 4- Feira da Pedreira; 5Feira do Telégrafo; 6- Feira do Ver-o-Peso.

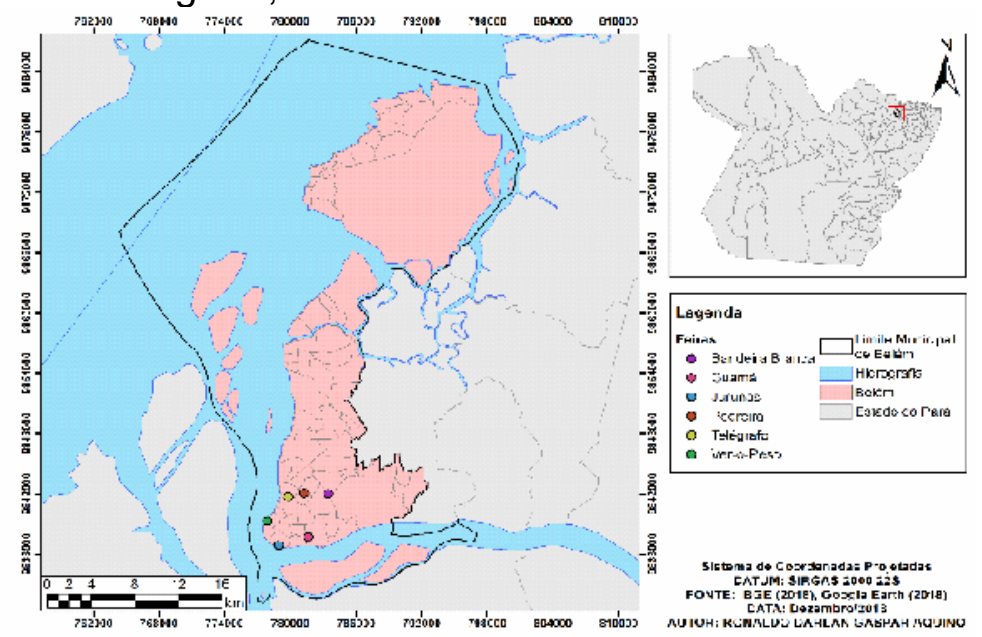

Fonte: Os autores (2021).

A coleta das garrafadas foi realizada nas principais feiras e mercados livres de Belém (Figura 1), sendo: a Feira da Bandeira Branca, Feira da Pedreira, Feira do Telégrafo, Feira do Jurunas e a Feira do Guamá, o Mercado do Ver-o-Peso e o Mercado de São Braz. Em um primeiro momento, foram coletadas 23 garrafadas das seis feiras mencionados. Sete garrafadas foram do Mercado do Ver-o-Peso, quatro da Feira da Pedreira, três da Feira da Bandeira Branca, três da Feira do Telégrafo, três da Feira do Jurunas e três da Feira do Guamá. Em seguida, estas foram levadas ao laboratório de pesquisa de Biodiversidade e Agroecologia do Programa de Pós-Graduação em Ciências Ambientais (PPGCA) da Universidade do Estado do Pará para fotografia e anotações dos rótulos de cada garrafa.

O estudo requereu uma abordagem qualitativa e descritiva, uma vez que analisou, observou, registrou e correlacionou aspectos que envolvem o conhecimento tradicional na prática das garrafadas com o conhecimento científico das plantas com poder medicinal sem manipulá-los. Foram estudados e revisados livros e artigos sobre medicina popular e o papel das garrafadas através do conhecimento popular, com a finalidade de aprofundar o conhecimento sobre o uso cultural e o potencial científico em 
discussão nessa pesquisa. Além disso, foram realizadas pesquisas em literaturas científicas especializadas na identificação e classificação de plantas.

Tendo como um dos objetivos específicos apresentar os nomes científicos de cada planta medicinal da composição das garrafadas, foram consultados o site Flora do Brasil, pois é referência em consulta de espécies para a flora brasileira (Disponível: < http://floradobrasil.jbrj.gov.br/>) e o site também de pesquisa científica na identificação de plantas, intitulado "Tropicos" (Disponível: <http://www.tropicos.org/Home.aspx>). Na busca por apresentar a composição química das plantas que se destacam pelo número de vezes que se repetiram nas garrafadas, o livro "Plantas Medicinais no Brasil: nativas e exóticas" de Harri Lorenzi e Matos (2008) foi consultado, sendo um dos livros de mais referência sobre plantas medicinais, o qual analisa não só características gerais das plantas, mas também aborda a etnobotânica e ensaios fitoquímicos e farmacológicos por outras literaturas.

\section{RESULTADOS E DISCUSSÃO}

Segundo estudos de Medeiros (2010), em relação à localização geográfica dos inúmeros pontos de feiras e mercados livres espalhados pela capital paraense, existem dois tipos de espaços de feiras: aqueles que se localizam a beira rio e aqueles que se espalham pelas ruas e avenidas de bairros centrais e periféricos. Ainda segundo autor, as feiras da Pedreira, Telégrafo, Jurunas e Guamá são feiras livres localizadas em bairros centrais e periféricos da capital paraense, e que levam o nome do bairro em que estão localizadas. Já a feira da Bandeira Branca está localizada no bairro do Marco, sendo uma das feiras mais antigas de Belém.

Das 23 garrafadas medicinais coletadas de feiras de Belém, foi montado o Quadro 1 listando as garrafadas e apresentando a composição de plantas medicinais, segundo descritos nos rótulos de cada garrafada. Foram descritos os nomes populares e o nome científico de cada espécie, além da indicação de uso de cada uma.

QUADRO 1. Composição de espécies botânicas das garrafadas analisadas nas feiras e mercados livres de Belém.

\begin{tabular}{|c|c|c|c|}
\hline Garrafada & $\begin{array}{l}\text { Planta Medicinal } \\
\text { (nome popular) }\end{array}$ & Nome Científico & Indicação de Uso \\
\hline \multirow{15}{*}{$\begin{array}{l}\text { Anti- } \\
\text { inflamatória }\end{array}$} & Unha de gato & Uncaria tomentosa (Willd. ex Schult.) DC. & \multirow[t]{15}{*}{ Inflamações em geral } \\
\hline & Uxi amarelo & Endopleura uchi (Huber) Cuatrec & \\
\hline & Casca de copaíba & Copaifera langsdorffii Desf. & \\
\hline & Jucá & Caesalpinia ferrea var. cearensis Huber & \\
\hline & Aroeira & Schinus terebinthifolia Raddi & \\
\hline & Barbatimão & Stryphnodendron adstringens (Mart.) Coville & \\
\hline & Feijão-guando & Cajanus cajan (L.) Huth & \\
\hline & Cajú & Anacardium occidentale L. & \\
\hline & Verônica & Dalbergia subcymosa Ducke & \\
\hline & Ipê roxo & Handroanthus impetiginosus (Mart. ex DC) Mattos & \\
\hline & Sucuuba & Himatanthus sp. Willdenow & \\
\hline & Flor de catingueira & Caesalpinia pyramidalis Tul. & \\
\hline & Cedro & Cedrus sp. (Roxb.) G. Don & \\
\hline & Andiroba & Carapa guianensis Aubl. & \\
\hline & Graviola & Annona muricata L. & \\
\hline Hemorroida & Raiz de & Polygala spectabilis DC. & Hemorróida, prisão de \\
\hline
\end{tabular}




\begin{tabular}{|c|c|c|c|}
\hline \multirow{6}{*}{ 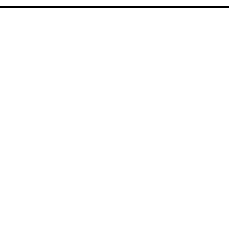 } & caamembeca & & \multirow[t]{6}{*}{ ventre e h. pylor } \\
\hline & Vassoura de botão & Borreria verticillata (L.) G.Mey. & \\
\hline & Aroeira & Schinus terebinthifolia Raddi & \\
\hline & Barbatimão & Stryphnodendron adstringens (Mart.) Coville & \\
\hline & Marupazinho & Eleutherine plicata (Sw.) Herb. & \\
\hline & Castanha da índia & Aesculus hippocastanum L. & \\
\hline \multirow{9}{*}{$\begin{array}{l}\text { Composto para } \\
\text { Diabetes }\end{array}$} & Folha de graviola & Annona muricata L. & \multirow[t]{9}{*}{ Tratamento de diabetes } \\
\hline & Caimbé & Coussapoa asperifólia Trécul & \\
\hline & Pata de vaca & Bauhinia forficata Link & \\
\hline & Açoite cavalo & Luehea divaricata Mart. \& Zucc. & \\
\hline & Carapanauba & Aspidosperma nitidum Benth. ex Müll.Arg. & \\
\hline & Miraruira & Salacia impressifolia (Miers.) A. C. Smith. & \\
\hline & Patchouli & Pogostemon patchouly Pell. & \\
\hline & Pau tenente & Quassia amara L. & \\
\hline & Folha de ajurú & Chrysobalanus icaco L. & \\
\hline \multirow{8}{*}{$\begin{array}{l}\text { Composto } \\
\text { para o Fígado }\end{array}$} & Boldo & Plectranthus barbatus (Coleus barbatus) Andrews & \multirow[t]{8}{*}{ Problemas de fígado } \\
\hline & Casca preciosa & Aniba canelilla (Kunth) Mez & \\
\hline & Cascara sagrada & Rhamnus purshiana D.C. & \\
\hline & Elixir-paregórico & Ocimum selloi Benth. & \\
\hline & Folha de Sucurijú & Mikania lindleyana D.C. & \\
\hline & Amor crescido & Portulaca pilosa L. & \\
\hline & $\begin{array}{l}\text { Casca de } \\
\text { carapanauba }\end{array}$ & Aspidosperma nitidum Benth. ex Müll.Arg. & \\
\hline & Casca de andiroba & Carapa guianensis Aubl. & \\
\hline \multirow{11}{*}{$\begin{array}{l}\text { Saúde da } \\
\text { Próstata }\end{array}$} & Uxi amarelo & Endopleura uchi (Huber) Cuatrec & \multirow{11}{*}{$\begin{array}{l}\text { Infecção das vias } \\
\text { urinárias, inflamação e } \\
\text { edema prostatal, sífilis, } \\
\text { gonorreia e debilidade } \\
\text { sexual. }\end{array}$} \\
\hline & Unha de gato & Uncaria tomentosa (Willd. ex Schult.) DC. & \\
\hline & Verônica & Dalbergia subcymosa Ducke & \\
\hline & Barbatimão & Stryphnodendron adstringens (Mart.) Coville & \\
\hline & Assacu & Hura crepitans L. & \\
\hline & Copaíba & Copaifera langsdorffii Desf. & \\
\hline & Açoite cavalo & Luehea divaricata Mart. \& Zucc. & \\
\hline & Cavalinha & Equisetum giganteum $\mathrm{L}$. & \\
\hline & Marapuama & Ptychopetalum uncinatum Anselmino & \\
\hline & Puxuri & Licaria puchury-major (Mart.) Kosterm. & \\
\hline & Noz moscada & Myristica fragrans Houtt & \\
\hline \multirow{5}{*}{$\begin{array}{l}\text { Preventivo do } \\
\text { Câncer }\end{array}$} & Extrato de assacu & Hura crepitans L. & \multirow{5}{*}{$\begin{array}{l}\text { Preventivo do câncer, } \\
\text { tratamento de miomas e } \\
\text { tumores cancerígenos e } \\
\text { infecções do útero e } \\
\text { ovário }\end{array}$} \\
\hline & Barbatimão & Stryphnodendron adstringens (Mart.) Coville & \\
\hline & Ipê roxo & Handroanthus impetiginosus (Mart. ex DC) Mattos & \\
\hline & Jucá & Caesalpinia ferrea var. cearensis Huber & \\
\hline & Aroeira & Schinus terebinthifolia Raddi & \\
\hline \multirow{10}{*}{$\begin{array}{l}\text { Suplemento } \\
\text { alimentar }\end{array}$} & Espinheira santa & Monteverdia ilicifolia Mart. & \multirow{10}{*}{$\begin{array}{l}\text { Gastrite, ulcera, pedra } \\
\text { nos rins, fígado, } \\
\text { hepatite, circulação, } \\
\text { ácido úrico, pressão } \\
\text { alta, varizes e } \\
\text { reumatismo }\end{array}$} \\
\hline & Alcachofra do Norte & Cynara cardunculus L. & \\
\hline & Artemísia & Artemisia vulgaris $\mathrm{L}$. & \\
\hline & Carqueja amarga & Baccharis trimera (Less.) DC. & \\
\hline & Castanha da índia & Aesculus hippocastanum L. & \\
\hline & Cavalinha & Equisetum giganteum $\mathrm{L}$. & \\
\hline & Chapéu de couro & $\begin{array}{l}\text { Echinodorus grandiflorus (Cham. \& Schltdl.) } \\
\text { Micheli }\end{array}$ & \\
\hline & Ipê roxo & Handroanthus impetiginosus (Mart. ex DC) Mattos & \\
\hline & Jurubeba & Solanum paniculatum $\mathrm{L}$. & \\
\hline & Salsaparrilha & Smilax papyracea Poir. & \\
\hline \multirow{4}{*}{$\begin{array}{l}\text { Xarope de } \\
\text { Cupim }\end{array}$} & Gengibre & Zingiber officinale Roscoe & \multirow[t]{4}{*}{ Tratamento de Gripes } \\
\hline & Sucupira & Pterodon emarginatus Vogel & \\
\hline & Urucu & Bixa orellana L. & \\
\hline & Alho & Allium sativum $\mathrm{L}$. & \\
\hline
\end{tabular}




\begin{tabular}{|c|c|c|c|}
\hline & Jatobá & Hymenaea courbaril L. & \\
\hline \multirow[t]{7}{*}{$\begin{array}{l}\text { Saúde dos } \\
\text { Pulmões }\end{array}$} & Leite de Amapá & $\begin{array}{l}\text { Parahancornia fasciculata (P. amapa) Ducke(Poir.) } \\
\text { Benoist }\end{array}$ & \multirow{7}{*}{$\begin{array}{l}\text { Pneumonia, efizema } \\
\text { pulmonar, tônico dos } \\
\text { pulmões, tosse brava, } \\
\text { anti-inflamatória, } \\
\text { bronquite, dor no peito, } \\
\text { falta de ar e } \\
\text { tuberculose }\end{array}$} \\
\hline & Jucá & Caesalpinia ferrea var. cearensis Huber & \\
\hline & Açafrão & Curcuma longa L. & \\
\hline & Erva de São João & Hypericum perforatum $\mathrm{L}$. & \\
\hline & Angico & Anadenanthera colubrina (Vell.) Brenan & \\
\hline & Jatobá & Hymenaea courbaril L. & \\
\hline & Verônica & Dalbergia subcymosa Ducke & \\
\hline \multirow{7}{*}{$\begin{array}{l}\text { Fígado } \\
\text { Saudável }\end{array}$} & Marcela & Achyrocline satureioides (Lam.) DC. & \multirow{7}{*}{$\begin{array}{l}\text { Tratamento de } \\
\text { estômago, fígado, azia, } \\
\text { má digestão, mal } \\
\text { hálito, diarreia e úlcera }\end{array}$} \\
\hline & Sucuuba & Himatanthus sp. Willdenow & \\
\hline & Folha do Pirarucu & Kalanchoe pinnata (Lam.) Pers. & \\
\hline & Sucurijú & Mikania lindleyana DC. & \\
\hline & Boldo & Plectranthus barbatus (Coleus barbatus) Andrews & \\
\hline & Babosa & Aloe vera (L.) Burman & \\
\hline & Amor crescido & Portulaca pilosa L. & \\
\hline \multirow[t]{3}{*}{ Quebra Pedra } & Cana do Brejo & Costus spicatus (Jacq.) Sw. & \multirow{3}{*}{$\begin{array}{l}\text { Elimina cálculos renais, } \\
\text { nefrites, cistites, } \\
\text { hepatite, enfermidades } \\
\text { crônicas da bexiga e } \\
\text { distúrbios da próstata }\end{array}$} \\
\hline & Quebra pedra & Phyllanthus spp. L. & \\
\hline & Chapéu de couro & $\begin{array}{l}\text { Echinodorus grandiflorus (Cham. \& Schltdl.) } \\
\text { Micheli }\end{array}$ & \\
\hline \multirow[t]{5}{*}{ Seca Barriga } & Feijão branco & Phaseolus sp L. & \multirow{5}{*}{$\begin{array}{l}\text { Combate a ansiedade, } \\
\text { gordura localizada, } \\
\text { celulite, colesterol, } \\
\text { prisão de ventre e } \\
\text { varizes }\end{array}$} \\
\hline & Banana verde & Musa spp. L. & \\
\hline & Soja preta & Glycine spp. Willd & \\
\hline & Maracujá & Passiflora edulis Sims & \\
\hline & Cenoura. & Daucus carota $\mathrm{L}$. & \\
\hline \multirow{9}{*}{$\begin{array}{l}\text { Saúde do } \\
\text { Útero }\end{array}$} & Aroeira & Schinus terebinthifolia Raddi & \multirow{9}{*}{$\begin{array}{l}\text { Inflamação do colo do } \\
\text { útero, nas trompas, } \\
\text { ovários e na vesícula, } \\
\text { menopausa, cisto e } \\
\text { miomas, dissolve e } \\
\text { destrói tumores, } \\
\text { hemorroida, regulariza } \\
\text { a menstruação }\end{array}$} \\
\hline & Jucá & Apuleia ferrea (Caesalpinia ferrea) (Benth.) Ducke & \\
\hline & Verônica & Dalbergia subcymosa Ducke & \\
\hline & Barbatimão & Stryphnodendron adstringens (Mart.) Coville & \\
\hline & Flor da catingueira & Caesalpinia pyramidalis Tul. & \\
\hline & Unha de gato & Uncaria tomentosa (Willd. ex Schult.) DC. & \\
\hline & Uxi amarelo & Endopleura uchi (Huber) Cuatrec & \\
\hline & Cajuí & Anacardium humile A. St.-Hil. & \\
\hline & Sucuuba & Himatanthus sp. Willdenow & \\
\hline \multirow{12}{*}{$\begin{array}{l}\text { Tratamento de } \\
\text { infecção }\end{array}$} & Barbatimão & Stryphnodendron adstringens (Mart.) Coville & \multirow{12}{*}{$\begin{array}{l}\text { Infecção, inflamação, } \\
\text { corrimento, coceira } \\
\text { vaginal, ferimento no } \\
\text { utero, dor de cólica, } \\
\text { tontura e dores no } \\
\text { corpo }\end{array}$} \\
\hline & Uxi amarelo & Endopleura uchi (Huber) Cuatrec & \\
\hline & Jucá & Caesalpinia ferrea var. cearensis Huber & \\
\hline & Ursina & Arctostaphylos uva-ursi (L.) Spreng. & \\
\hline & Folha santa & Bryophyllum calycinum Salisb. & \\
\hline & Saboeira & Saponaria officinalis $\mathrm{L}$. & \\
\hline & Quinquina & Quinquina officinalis (L.) Kuntze & \\
\hline & Aroeira & Schinus terebinthifolia Raddi & \\
\hline & Catuaba & $\begin{array}{l}\text { Anemopaegma arvense (Vell.) Stellfeld ex de } \\
\text { Souza }\end{array}$ & \\
\hline & Pau ferro & Libidibia ferrea (Mart. ex Tul.) L.P.Queiroz & \\
\hline & Angico & Anadenanthera spp. Speg. & \\
\hline & jurubeba & Solanum paniculatum $\mathrm{L}$. & \\
\hline \multirow[t]{5}{*}{ Menopausa } & Noni & Morinda citrifolia L. & \multirow{5}{*}{$\begin{array}{l}\text { Alivia os sintomas } \\
\text { desconfortáveis } \\
\text { provocados pela } \\
\text { menopausa } \\
\text { andropausa, como: mal }\end{array}$} \\
\hline & Amora & Morus sp. L. & \\
\hline & Erva doce & Pimpinella anisum L. & \\
\hline & Camomila & Matricaria recutita L. & \\
\hline & Erva cidreira & Melissa officinalis L. & \\
\hline
\end{tabular}




\begin{tabular}{|c|c|c|c|}
\hline & Boldo & Plectranthus barbatus (Coleus barbatus) Andrews & $\begin{array}{l}\text { humor, irritação, dores } \\
\text { de cabeça e calor }\end{array}$ \\
\hline \multirow{3}{*}{$\begin{array}{l}\text { Elixir da flor da } \\
\text { Catingueira }\end{array}$} & Flor da catingueira & Caesalpinia pyramidalis Tul. & \multirow{3}{*}{$\begin{array}{l}\text { Estômago, arroto } \\
\text { frequente, azia, boca } \\
\text { amarga, ventosidade } \\
\text { na barriga e enjoos }\end{array}$} \\
\hline & Marcela & Achyrocline satureioides (Lam.) DC. & \\
\hline & Amor crescido & Portulaca pilosa L. & \\
\hline \multirow[t]{4}{*}{ Anti-reumático } & Mururé & Pistia stratiotes L. & \multirow{4}{*}{$\begin{array}{l}\text { Agente diurético, } \\
\text { depurativo, sudorifico, } \\
\text { anti-inflamatório } \\
\text { antirreumático; } \\
\text { eficientes em } \\
\text { reumatismo crônico, } \\
\text { artrite e dores nas } \\
\text { juntas }\end{array}$} \\
\hline & Chapéu de couro & $\begin{array}{l}\text { Echinodorus grandiflorus (Cham. \& Schltdl.) } \\
\text { Micheli }\end{array}$ & \\
\hline & Salsaparrilha & Smilax papyracea Poir. & \\
\hline & Unha de gato & Uncaria tomentosa (Willd. ex Schult.) DC. & \\
\hline \multirow[t]{2}{*}{ Anti-colesterol } & Berinjela & Solanum melongena L. & \multirow{2}{*}{$\begin{array}{l}\text { Coadjuvante nas dietas } \\
\text { de emagrecimento, } \\
\text { hipertensão, hidropisia } \\
\text { e arteriosclerose. }\end{array}$} \\
\hline & Alcachofra & Cynara scolymus L. & \\
\hline \multirow{5}{*}{$\begin{array}{l}\text { Saúde da } \\
\text { Mulher }\end{array}$} & Barbatimão & Stryphnodendron adstringens (Mart.) Coville & \multirow{5}{*}{$\begin{array}{l}\text { Regula a menstruação, } \\
\text { combate corrimento e } \\
\text { inflamação no útero }\end{array}$} \\
\hline & Ipê roxo & Handroanthus impetiginosus (Mart. ex DC) Mattos & \\
\hline & Jucá & Caesalpinia ferrea var. cearensis Huber & \\
\hline & Verônica & Dalbergia subcymosa Ducke & \\
\hline & Unha de gato & Uncaria tomentosa (Willd. ex Schult.) DC. & \\
\hline \multirow[t]{6}{*}{ Elixir Gastrite } & Boldo & Plectranthus barbatus (Coleus barbatus) Andrews & \multirow{6}{*}{$\begin{array}{l}\text { Tratamento de gastrite, } \\
\text { úlcera, bom para } \\
\text { cólicas } \\
\text { e doenças do } \\
\text { estômago }\end{array}$} \\
\hline & Carqueja amarga & Baccharis trimera (Less.) DC. & \\
\hline & Espinheira santa & Monteverdia ilicifolia Mart. & \\
\hline & Malva & Malva spp. L. & \\
\hline & Ipê roxo & Handroanthus impetiginosus (Mart. ex DC) Mattos & \\
\hline & Cascára sagrada & Rhamnus purshiana D.C. & \\
\hline \multirow{5}{*}{$\begin{array}{l}\text { Elixir anti- } \\
\text { anêmico }\end{array}$} & Noni & Morinda citrifolia L. & \multirow{5}{*}{$\begin{array}{l}\text { Falta de ar, cansaço, } \\
\text { falta de energia, dor no } \\
\text { peito, palidez na pele, } \\
\text { câimbra, hipertensão, } \\
\text { sonolência e tontura }\end{array}$} \\
\hline & Jucá & Caesalpinia ferrea var. cearensis Huber & \\
\hline & Pariri & Arrabidaea chica (Humb. \& Bonpl.) B. Verl. & \\
\hline & Sucuuba & Himatanthus sp. Willdenow & \\
\hline & Caxinguba & Ficus insipida Willd. & \\
\hline \multirow[t]{5}{*}{$\begin{array}{l}\text { Batatão } \\
\text { Hipólito }\end{array}$} & Batata de purga & $\begin{array}{l}\text { Operculina hamiltonii (G. Don) D.F. Austin \& } \\
\text { Staples }\end{array}$ & \multirow{5}{*}{$\begin{array}{l}\text { Indicado nas gestões } \\
\text { cerebrais, derrame, } \\
\text { convulsão, paralisia } \\
\text { facial, boca torta, } \\
\text { epilepsia e má } \\
\text { circulação do sangue }\end{array}$} \\
\hline & Pixuri & Licaria puchury-major (Mart.) Kosterm. & \\
\hline & Noz moscada & Myristica fragrans Houtt & \\
\hline & Carqueja amarga & Baccharis trimera (Less.) DC. & \\
\hline & Flor da catingueira & Caesalpinia pyramidalis Tul. & \\
\hline \multirow{9}{*}{$\begin{array}{l}\text { Inflamação e } \\
\text { câncer }\end{array}$} & Verônica & Dalbergia subcymosa Ducke & \multirow{9}{*}{$\begin{array}{l}\text { Indicado para o } \\
\text { tratamento de todos os } \\
\text { tipos de câncer e } \\
\text { inflamações }\end{array}$} \\
\hline & Aroeira & Schinus terebinthifolia Raddi & \\
\hline & Sucuuba & Himatanthus sp. Willdenow & \\
\hline & Barbatimão & Stryphnodendron adstringens (Mart.) Coville & \\
\hline & Pau darco roxo & Handroanthus heptaphyllus (Vell.) Mattos & \\
\hline & Casca de caju & Anacardium occidentale L. & \\
\hline & Unha de gato & Uncaria tomentosa (Willd. ex Schult.) DC. & \\
\hline & $\begin{array}{l}\text { Casca de uxi } \\
\text { amarelo }\end{array}$ & Endopleura uchi (Huber) Cuatrec & \\
\hline & Fruta cabeluda & $\begin{array}{l}\text { Myrciaria glazioviana (Kiaersk.) G.M.Barroso ex } \\
\text { Sobral }\end{array}$ & \\
\hline
\end{tabular}

Fonte: Os autores (2021). 
O quadro acima apresenta o nome de cada garrafada, todas comercializadas para fins medicinais. De acordo com o Quadro 1, algumas das garrafadas podem chegar a ter em sua composição de duas a 15 espécies de plantas. Como exemplo, a "Garrafada Anti-inflamatória", que possui a combinação de 15 plantas. Em contrapartida, a "Garrafada Anticolesterol" possui a combinação de apenas duas plantas, a berinjela e a alcachofra.

As plantas destacadas no quadro 1, como a Jucá e o Barbatimão, são plantas encontradas em mais de cinco garrafadas medicinais das 23 estudadas. Isso se deve às suas propriedades bioquímicas e farmacobotânicas. Também foi abordado o nome científico e autor de cada espécie botânica, uma vez que o uso das garrafadas são de cunho cultural e vendidas de forma informal nas feiras e mercados. Os rótulos dessas garrafas apresentam a composição de plantas com seus nomes comuns, vernacular ou ainda vulgar.

Os indicativos de uso das garrafadas estão apresentados no quadro 1, conforme nos seus respectivos rótulos. Foi possível observar que mais de $95 \%$ das garrafadas analisadas, eram para uso de tratamento de doenças ou outros males, como a garrafada "Fígado Saudável", indicada para o tratamento de estômago, fígado, azia, má digestão, mal hálito, diarreia e úlcera. Pouco mais de $4 \%$ para a prevenção de uma doença, que é o caldo da garrafada "Preventivo do Câncer", que como o nome sugere é indicada para prevenir câncer, mantendo o organismo saudável e o metabolismo controlado, para que funcione da forma correta, diminuindo a probabilidade de um possível descontrole metabólico.

A Annona muricata L. (graviola), presente na garrafada "anti-inflamatória" e em outras, possui diversas utilizações populares e está relacionada com cada parte da planta: folha, semente e casca (INDRAS, 2017). A garrafada "Composto para diabetes" utiliza as folhas da planta na mistura para o tratamento de diabetes. Porém, a eficácia e a segurança das preparações populares com $A$. muricata ainda não foram comprovadas cientificamente (LORENZI; MATOS, 2008).

Além da $A$. muricata, outras plantas são comumente encontradas nas preparações populares como as garrafadas. Estudos de Indras (2017) alertam que muitas plantas são tóxicas e podem causar problemas graves, como reações alérgicas, disfunções gastrintestinais, neurológicas, hepáticas, hematológicas e renais, podendo até mesmo levar à morte. Assim, é evidente a importância de estudos de misturas vegetais nos compostos populares medicinais para identificação de possíveis efeitos das propriedades dessas plantas no organismo. Merecem também atenção os rótulos em que estão os indicativos das garrafadas pois muitos não possuem contra indicação do uso (PASSOS et al., 2018).

Foram identificadas 47 famílias nas garrafadas estudadas. A família botânica com maior número de espécies dentro das 23 garrafadas analisadas foi a Fabaceae, com 14 espécies. Em seguida a Asteraceae, com cinco espécies, e as famílias Rubiaceae e Bignoniaceae com quatro espécies cada. As demais famílias possuem apenas duas ou uma única espécie representativa. Segundo Lima (2000), além de a terceira maior família das Angiospermas do mundo, a Fabaceae é considerada a maior família no Brasil, com 2.100 espécies e 188 gêneros, dos quais 31 são endêmicos, estando representada em todos os biomas brasileiros. O quadro 2 apresenta plantas 
que se destacaram por estarem presentes em mais de cinco garrafadas dentre as 23 estudadas.

O metabolismo secundário vegetal, através das substâncias formadas no metabolismo primário, forma vários compostos orgânicos; que por sua vez possuem atividade biológica. As principais classes de metabólitos secundários identificados em espécies vegetais são os compostos nitrogenados, compostos fenólicos ou fenóis e terpenos ou terpenóides. (DELBONE; LANDO, 2010). Segundo Angelo e Jorge (2007), os principais compostos fenólicos e mais abundantes antioxidantes naturais são os flavonoides, ácidos fenólicos, taninos e tocoferóis. Os flavonoides estão presentes em frutas, folhas, sementes e em outras partes dos vegetais em forma de glicosídios. A classe dos compostos fenólicos tem vários grupos, um deles dão os taninos que estão divididos em duas classes: taninos hidrolisáveis e condensados. Os tocoferóis, outro dos fenólicos, estão presentes em vegetais, principalmente em sementes oleaginosas e folhas.

$\mathrm{Na}$ classe de polifenóis existem as catequinas, que possui como benefícios a saúde humana, a redução do surgimento de certos tipos de câncer, redução do colesterol sérico e estimulação do sistema imunológico (PEREIRA; CARDOSO, 2012). Derivados também do metabolismo secundário tem-se as saponinas que apresentam propriedades detergentes e surfactantes. Seu efeito biológico destaca-se pela ação antioxidante, em que se ligam a sais biliares e colesterol no tubo digestivo; além disso atuam contra células tumorais (PEREIRA; CARDOSO, 2012).

QUADRO 2. Plantas com maior frequência nas garrafadas e seus metabólitos secundários segundo Lorenzi (2002) "Plantas Medicinais no Brasil: nativas e exóticas".

\begin{tabular}{|c|c|c|c|}
\hline & Nome Científico & $\begin{array}{l}\text { Metabólitos com atividade } \\
\text { biológica }\end{array}$ & Uso etnobotânico \\
\hline Aroeira & $\begin{array}{l}\text { Schinus } \\
\text { terebinthifolia } \\
\text { Raddi }\end{array}$ & $\begin{array}{l}\text { Tanino } \\
\text { Flavonoides } \\
\text { Ácidos triterpênico } \\
\text { Mono e sequiterpenos } \\
\text { Alquil-fenóis }\end{array}$ & $\begin{array}{l}\text { Uso das cascas cozidas por vários dias, } \\
\text { em banhos de assento após o parto } \\
\text { como anti-inflamatório e cicatrizante. As } \\
\text { folhas e frutos são adicionados à água } \\
\text { de lavagem de feridas e úlceras } \\
\text { (LORENZI, 2002; BRAGA, 1960; } \\
\text { GUENWALD, BRENDLER, JAENICKKE, } \\
\text { 2000). }\end{array}$ \\
\hline Barbatimão & $\begin{array}{l}\text { Stryphnodendron } \\
\text { adstringens } \\
\text { (Mart.) Coville }\end{array}$ & $\begin{array}{l}\text { Substâncias tânicas } \\
\text { Mucilagens } \\
\text { Flavonoides } \\
\text { Corantes vermelho } \\
\text { Açúcar solúvel } \\
\text { Alcaloides não } \\
\text { determinados }\end{array}$ & $\begin{array}{l}\text { Chá da casca em uso externo de males. } \\
\text { Casca risca em tanino de grande ação } \\
\text { estíptica, é empregada na indústria de } \\
\text { curtume (PANIZZA, S. 1998). }\end{array}$ \\
\hline Jucá & $\begin{array}{l}\text { Caesalpinia } \\
\text { ferrea var. } \\
\text { cearensis Huber }\end{array}$ & $\begin{array}{l}\text { Derivados de forbol (TPA) } \\
\text { Compostos antracênicos } \\
\text { Derivados do ácido elágico } \\
\text { Derivado trihidroxilado da } \\
\text { acetofenona }\end{array}$ & $\begin{array}{l}\text { "Tintura-da-bage-de-jucá" forma um } \\
\text { xarope muito popular no Nordeste para } \\
\text { tratamento caseiro de tosse, bronquite e } \\
\text { coqueluche (MATOS, F.J.A. 1997). }\end{array}$ \\
\hline $\begin{array}{l}\text { Unha de } \\
\text { gato }\end{array}$ & $\begin{array}{l}\text { Uncaria } \\
\text { tomentosa (Willd. } \\
\text { ex Schult.) DC. }\end{array}$ & $\begin{array}{l}\text { Alcaloides oxindólicos } \\
\text { Glicosídeos do ácido } \\
\text { quinóvico } \\
\text { Taninos }\end{array}$ & $\begin{array}{l}\text { Chá dos ramos mais finos para o } \\
\text { tratamento de disenteria (SCHULTES, } \\
\text { R.E. \& RAFFAUF, R.F. 1990; UPHOF, } \\
\text { R.C.R. 1968). Além de empregarem }\end{array}$ \\
\hline
\end{tabular}




\begin{tabular}{|l|l|l|}
\hline & Catequinas & outras partes da planta para o \\
& Polifenóis & tratamento de uma ampla gama de \\
& Procianidinas A, B1, B2 e & moléstias (OCAMPO, T.P. 1994). \\
& Esteróis & \\
\hline
\end{tabular}

Fonte: Os autores (2021).

A S. Adstringens se mostrou presente nas garrafadas "Anti-inflamatória", "Hemorroida", "Saúde da Próstata", "Preventivo do Câncer", "Saúde do Útero", "Tratamento de infecção", "Saúde da Mulher" e "Inflamação e câncer". A espécie é conhecida popularmente como "barbatimão", ou ainda, "casca da virgindade", "abaramoteno", "charãozinho-roxo" e outros, dependendo da região em que se encontra (LORENZI, 2002). A planta é altamente empregada na medicina caseira e popular em grande parte das regiões do País.

Segundo estudos de Lorenzi (2002), a casca da S. Adstringens é empregada na indústria de curtume, tintura da casca, corante vermelho, e é utilizada também pelas indústrias para produzir tinta de escrever. A espécie ainda é rica em taninos, que são compostos do metabolismo secundário vegetal ou metabolismo especial importantes nas interações entre a planta e seu ecossistema (CASTEJON, 2011). São encontrados nos grupos de fenóis vegetais, fazendo com que sejam altamente reativos quimicamente.

Castejon (2011) também discute em seu estudo o mecanismo de atividade antioxidante do barbatimão. Essa atividade é atribuída também aos flavonoides, além dos taninos, que auxiliam no processo de cura, já que os radicais livres são um fator importante na formação de lesões ulcerativas e erosivas do trato gastrintestinal (BORRELLI; IZZO, 2000; CARBONEZI et al., 2007). Hermenegildo (2016) reforça que por ser um elemento antioxidante, o tanino faz um grande bem à saúde. É possível observar o válido emprego do barbatimão nas garrafadas indicadas para uso de tratamentos de inflamações. Como as garrafadas "Saúde do Útero" e "Saúde da Mulher", onde são indicadas para inflamação do colo do útero.

A $U$. Tomentosa e a $S$. terebinthifolia também apresentam compostos taninos, o que Ihes conferem propriedades anti-inflamatórias, características da maioria das garrafadas estudadas. Ocampo (1994) analisou o uso da U. Tomentosa por indígenas no tratamento de várias doenças, como asma, artrite, reumatismo e para curar ferimentos profundos. Outros estudos etnobotânicos revelaram o uso do chá dos ramos da planta no Amazonas, para tratamento de desinteria (SCHULTES; RAFFAUF, 1990).

Lorenzi (2008) trouxe em seu livro pesquisas de Matta et al. (1976) e Keplinger et al. (1990) em que analisaram a presença nas raízes e cascas da $U$. Tomentosa de alcaloides oxindólicos e estudos relatando o poder de estimular o sistema imunológico em até $50 \%$. Isso induziu o emprego da planta no tratamento de doenças que afetam o sistema imunológico, e na garrafada "Inflamação e câncer" é notada a presença da planta em sua composição no emprego do tratamento de todos os tipos de cânceres.

Os glicosídeos do ácido quinóvico estão sendo discutidos também por cientistas em ensaios fitoquímicos no tratamento anti-inflamatório e antiviral (JÜRGENSEN, 2007). Os compostos antioxidantes (taninos, catequinas, polifenóis e procianidinas $A$, $\mathrm{B} 1, \mathrm{~B} 2$ e B4), assim como os esteróis da planta estão também ligados às suas propriedades anti-inflamatórias. Uma classe de compostos conhecidos, os fitosteróis (b- 
sitosterol, estigmastelrol, campesterol isolados), que são encontrados na "unha de gato", nome popular da espécie, são documentados com propriedades imunoestimulante, anti-inflamatória, anticancerígena e reparadora das células (ALONSO, 2004). Isso reforça o valor científico e explica o emprego dessa planta nas garrafadas.

A S. Terebinthifolia, popularmente é conhecida por "aroeira", ou aroeira-da-praia, aroeira-precoce, aroeira-mansa, aroeira-vermelha, aroeira-pimenteira, aroeira-do-brejo, aroeira-negra, aroeira-branca, aroeira-do-campo e outros, dependendo da região em que se encontra (LORENZI; MATOS, 2008). A literatura etnobotânica cita o uso da casca na forma de cozimento por mulheres após o parto como anti-inflamatória e cicatrizante. Além dos compostos taninos presentes na planta, apresentam também flavonoides e ácidos triterpênicos nas cascas e de até $5 \%$ de óleo essencial formado por mono e sequiterpenos nos frutos e nas folhas (LORENZI; MATOS 2008). Estudo fitoquímico e biológico realizados em $S$. Terebinthifolia descreveram a ocorrência de terpenóides e ácidos graxos também em sua constituição (FALCÃO et al., 2015).

Um ensaio clínico feito com extrato aquoso das cascas da $S$. Terebinthifolia, na concentração de $10 \%$, aplicado na forma de compressas intravaginais em 100 mulheres portadoras de cervicite e cervicovaginites, provou $100 \%$ de cura num período de uma a três semanas de tratamento (BANDEIRA; WANICK, 1974; LORENZI; MATOS, 2008). Nas garrafadas "Preventivo do Câncer", "Saúde do Útero" e "Tratamento de infecção", a planta é indicada para o tratamento de infecção e inflamação do colo do útero, o que mostra o potencial científico dessas garrafadas.

Outra espécie com destaque no quadro 2 é a $C$. Ferrea, chamada porpulamente de "jucá", mas conhecida nas diversas regiões de ibirá-obi ou baje-de-jucá. É usada como um antigo costume na medicina popular no Nordeste como tintura, e também como xarope no tratamento caseiro de tosse (MATOS, 1997). Estudos recentes farmacológicos do estrato de hidroalcoólico do fruto da espécie comprovaram sua ação contra tumores provocados pelo vírus Epstein-Barr, além de atividade inibidora da formação de papilomas induzidos na pele de ratos por ação dos derivados do forbol (TPA) e de componentes antracênicos (NAKAMURA et al., 2002).

Em trabalhos anteriores, mostraram atividade hiperglicemiante atribuídas aos derivados do ácido elágico e seu derivado trihidroxilado e uma potente atividade inibidora dos tumores, demonstradas in vitro, relacionadas com três derivados trihidroxilado da acetofenona (CARVALHO et al., 1996; NAKAMURA et al., 2002; LORENZI; MATOS, 2008). Não foi atribuído o emprego da C. Ferrea em garrafadas indicadas para tratamento hiperglicemiante.

\section{CONCLUSÃO}

A partir dos resultados obtidos, foi possível constatar que as análises das garrafadas revelaram dados curiosos a respeito da quantidade e variedades de espécies botânicas presente dentro de uma única garrafada, podendo apresentar de duas a 15 plantas em sua mistura. Além disso, observou-se uma diversidade de espécies botânicas importantes não só na medicina popular e caseira, mas também no seu valor científico, na identificação de metabólitos com atividades biológicas descritas na literatura científica de espécies com alto potencial científico. 
O emprego eficaz e seguro das plantas medicinais em preparações caseiras e popularmente comercializadas deve ser estimulado, uma vez que se evidenciou no presente artigo o valor científico das garrafadas através do potencial de plantas com propriedades medicinais. Porém, o uso dessas plantas nos preparos caseiros, como garrafadas, deve ser com atenção e cuidado ao manipulá-las. É importante destacar o quão é essencial o estudo de extratos vegetais para identificação de substâncias bioativas e suas aplicações na medicina popular.

\section{REFERÊNCIAS}

ANGELO, P. M.; JORGE, N. Compostos fenólicos em alimentos - uma breve revisão. Revista do Instituto Adolfo Lutz, v. 66, n.1, São Paulo, 2007.

ALONSO, J. Tratado de Fitofármacos e Nutracéuticos. Rosario: Corpus, 2004.

BANDEIRA, F. J. A.; WANICK, M. C. 1974. Ação anti-inflamatória e cicatrizante de Schinus aroeira Vell., em pacientes com cervicite e cervogaginite. Revista do Instituto de estudos Brasileiros, Antibióticos, Recife, p. 105-106. 1974.

BORRELLI, F.; IZZO, A. A. The plant kingdom as a source of anti-ulcer remedies. Phytotherapy Research [online], v.14, n.8, p.1099-1573, 2000. Disponível em: http://dx.doi.org/10.1002/1099573(200012)14:8<581::AIDPTR776>3.0.CO;2-S. Acesso em: $15 / 12 / 2018$.

BRASIL, Lei № 13.123, de 20 de maio de 2015. Dispõe sobre o acesso ao Patrimônio Genético, sobre a proteção e o acesso ao Conhecimento Tradicional associado e sobre a Repartição de Benefícios para conservação e uso sustentável da biodiversidade: Revoga a Medida Provisória no 2.186-16, de 23 de agosto de 2001; e dá outras providias. Diário Oficial da república Federativa do Brasil - Brasília, 20 de maio de 2015.

BRASIL - Ministério da Saúde. Secretaria de Ciência, Tecnologia e Insumos Estratégicos. Departamento de Assistência Farmacêutica. Política nacional de plantas medicinal e fitoterápico / Ministério da Saúde, Secretaria de Ciência, Tecnologia e Insumos Estratégicos, Departamento de Assistência Farmacêutica. - Brasília: Ministério da Saúde, 2006.

BRASIL, Lei № 5.991, de 19 de dezembro de 1973. Dispõe sobre o Controle Sanitário do Comércio de Drogas, Medicamentos, Insumos Farmacêuticos e Correlatos, e dá outras Providências. Diário Oficial da república Federativa do Brasil - Brasília, 19 de dezembro de 1973.

ANVISA - Agência Nacional de Vigilância Sanitária. Resolução de Diretoria Colegiada no. 48 de 16 de março de 2004. Aprova o regulamento técnico de medicamentos fitoterápico junto ao Sistema Nacional de Vigilância Sanitária. DOU. Diário Oficial da União, Poder Executivo - Brasília, 18 março de 2004. 
CAMARGO, M. T.L. A. A garrafada na medicina popular: uma revisão historiográfica. Dominguezia. Buenos Aires, p. 41-49, 2011.

CARVALHO, J. C., TEIXEIRA, J. R., SOUZA, P. J., BASTOS, J. K., DOS SANTOS FILHO, D., \& SARTI, S. J., 1996. Preliminary studies of analgesic and anti-inflammatory properties of Caesalpinia ferrea crude extract. Journal of ethnopharmacology, 53(3), 175-178. https://doi.org/10.1016/0378-8741(96)01441-9

CARBONEZI, C. A.; HAMERSKI, L.; GUNATILAKA, A. A.L., CAVALHEIRO, A.; CASTRO-GAMBOA, I.; SILVA, D.H.S. Bioactive flavone dimers from Ouratea multiflora (Ochnaceae). Revista brasileira de farmacognosia. [online], v.17, n.3, p.319-324, 2007.

Disponível em:http://www.scielo.br/scielo.php?script=sci_arttext\&pid=S0102695X2007000300003\&l ng=pt\&nrm=iso. Acesso em: 03 set. 2011.

CASTEJON, F. V., 2011. Taninos e Saponinas. Universidade Federal de Goiás, Goiânia, 2011.

DELBONE, C. A.C.; LANDO, R. L. Importância ecológica e evolutiva dos principais grupos de metabólitos secundários nas espécies vegetais.Congresso de Educação do Norte Pioneiro. 10ª edição. UENP-CCNE-CLA-Campus Jacarezinho. 2010. ISSN-1808-3579.

FALCÃO, M. P. M. M.; OLIVEIRA, T. K. B. de; SARMENTO, D. de A.; RODRIGUES, N. P. do Ó; GADELHA, N. C., 2015. Schinus terebinthifolius Raddi (Aroeira) e suas propriedades na Medicina Popular. Revista Verde (Pombal - PB - Brasil), v.10, n. 5 (ESPECIAL), p. 23 - 27, Dez/ 2015.

FRANÇA, I. S. S. X. De; SOUZA, J. A. De; BAPTISTA, R. S.; BRITTO, V. R. de S., 2007. Medicina popular: benefícios e malefícios das plantas medicinais Medicina popular: benefícios e malefícios das plantas medicinais. Revista Brasileira de Enfermagem, Brasília,mar/abr; 61(2): 201-8. 2.2008.

HERMENEGILDO, B. Entenda o que são taninos, onde estão e para que servem. Blog. Disponível em: < https://blog.artdescaves.com.br/o-que-sao-taninos-onde-estaopara-que-servem > Acesso em 13/03/2019.

IBGE - Instituto Brasileiro de Geografia e Estatística - Brasil/Pará/Belém/ 2010| v.4.3.14.0. 2010 .

INDRAS, D. M. Estudo da toxicidade de garrafada de uso popular. 2017. $84 \mathrm{f}$. Dissertação (Mestrado) - Universidade Estadual do Oeste do Paraná, Cascavel, 2017. Disponível em: http://tede.unioeste.br/handle/tede/2957.

JÜRGENSEN, S. Estudo dos mecanismos envolvidos no efeito antinociceptivo de uma fração de alcalóides oxindólicos de Uncaria tomentosa. Florianópolis, 2007. 
Xp. Dissertação (Mestrado em Farmacologia) - Curso de Pós-Graduação em Farmacologia, Universidade Federal de Santa Catarina.

KEPLINGER, K.; WAGNER, H.; KREUTZKAMP, B. Oxindole alkaloids having properties stimulating the immunologic system and preparation containing the same. US n. PI 940725, jul/1990.

LIMA, H. C. 2000. Leguminosas arbóreas da Mata Atlântica: uma análise da riqueza, padrões de distribuição geográfica e similaridades florísticas em remanescentes florestais do Estado do Rio de Janeiro. Tese - Universidade Federal do Rio de Janeiro, Rio de Janeiro, 2000.

LORENZI, H. Árvores brasileiras: manual de identificação e cultivo de plantas arbóreas nativas do Brasil. Nova Odessa, SP: Instituto Plantarum, 2002, 370 p.

LORENZI, H; MATOS, F. J. A. Plantas Medicinais no Brasil: nativas e exóticas. 2. Ed, Nova Odessa, SP: Instituto Plantarum, pp. 67-288, 2008.

MATTA, S. M., MONACHE, F. D., FERRARI, F., MARINI-BETTOLO, G. B. Alkaloids and procyanidins of an Uncaria sp. from Peru. II Farmaco; Edizione Scientifica. Jul;31(7):527-535.1976.

MATOS, F. J. A. 1997. O formulário fitoterápico do professor Dias da Rocha. 2.ed. Edição UFC, Fortaleza, 258 p.

MEDEIROS, J. F. da S. As feiras livres em Belém (Pa). Dissertação - Universidade Federal do Pará, Instituto de Filosofia e Ciências Humanas, Programa de PósGraduação em Geografia, Belém, 2010.

NAKAMURA, E. S., KUROSAKI, F., ARISAWA, M., MUKAINAKA, T., OKUDA, M., et al., Cancer chemopreventive effects of constituents of Caesalpinia ferrea and related compounds. Cancer letters, 177(2), 119-124.2002. https://doi.org/10.1016/s03043835(01)00708-x.

OCAMPO, T. P.; Uncaria tomentosa, Aspenctos Ethnomédicos, Médicos, Farmacológicos, Botânicos, Agronômicos, Comerciales, Legales, Anthropológicos, Sociales y Políticos. Instituto de Desarrollo Rural Peruano (IDDERP), Lima, 1994, 74 p.

PREFEITURA DE BELÉM, 2017. Coordenadoria Municipal de Turismo do município de Belém (BELEMTUR). Disponível: <http://www.belem.pa.gov.br/belemtur/site/index.php>. Acesso em: 10 jan/ 2019.

PASSOS, M. M. B.; ALBINO, R. da C.; SILVA, M. F.; OLIVEIRA, D. R., 2018. The cultural dissemination of garrafadas in Brazil: a parallel between popular medicine and 
sanitary legislation. Saúde Debate. Rio de Janeiro, v. 42, n.116, p. 248-262, Jan/mar 2018. DOI: 10.1590/0103-1104201811620.

PEREIRA, R. J.; CARDOSO, M. G. Metabólitos secundários vegetais e benefícios antioxidantes. Journal of Biotechnology and Biodiversity, v.. 3, n. 4: p. 146-152. 2012. ISSN-2179-4804.

SCHULTES, R. E.; RAFFAUF, R. F.; The Healing Foret - Medicinal and Toxic Plants of the Northwest Amazonia. Dioscorides Press, Portland, OR, 1990, 484 p.

SOUZA, R. Y. F.; Garrafada: o saber popular e a abordagem CTS. Trabalho de conclusão de curso - Universidade de Brasília/ Instituto de Química. Brasília, 2011. 